\title{
Uma Arquitetura Pedagógica para Aprendizagem de Pilotagem de Aeronaves
}

\author{
Edgar Eler, Orivaldo de Lira Tavares \\ Laboratório de Informática na Educação \\ Universidade Federal do Espírito Santo (UFES) - Vitória, ES - Brasil \\ elerdedgar.systems, tavares@inf.ufes.br
}

\begin{abstract}
The aircraft piloting activity requires a high level of preparation from the pilots, which in most cases results in an extensive period of theoretical and practical training by the apprentice. Through the use of flight simulators, the apprentices establish the first contacts with the piloting environment still on the ground, directly on computer, reducing the training costs and increasing safety. However, over the years, developments in simulators has been focused on operational accuracy and visual quality, excluding pedagogical aspects of pilot training. This paper proposes a pedagogical architecture for aircraft piloting learning, in which the practice in flight simulators is assisted by synthetic agents who question the student about his decisions and make him reflect on the most important decisions, explain the reasons that led him to take such decisions and examine other possible alternatives.
\end{abstract}

Key words: Learning Assistant, Aircraft Pilot Training Support, MultiAgent System, Flight Simulation.

Resumo. A pilotagem de aeronaves requer um alto nivel de preparação dos pilotos, o que, em geral, se reflete em um periodo extensivo de formação teórica e prática dos aprendizes. Por meio da utilização dos simuladores de voo, os aprendizes estabelecem os primeiros contatos com o ambiente de pilotagem ainda em terra, diretamente no computador, reduzindo os custos de treinamento e aumentando a segurança. Porém, ao longo dos anos, a evolução nos simuladores foi focada na precisão operacional e na qualidade visual, sem incluir aspectos pedagógicos na aprendizagem da pilotagem. Este artigo propõe uma arquitetura pedagógica para a aprendizagem de pilotagem de aeronaves, em que a prática em simuladores de voo seja assistida por agentes sintéticos que questionam o aprendiz sobre as decisões dele e fazem-no refletir sobre as decisões mais importantes, explicar os motivos que o fizeram tomar essas decisões e analisar outras possíveis alternativas.

Palavras-chave: Assistente de Aprendizagem, Apoio à Formação de Piloto de Aeronave, Sistema Multiagente, Simulação de Voo.

\section{Introdução}

A formação de indivíduos para exercerem a pilotagem de aeronaves foi aprimorada nas últimas décadas com a utilização dos simuladores, sistemas computacionais que 
simulam, de forma visual e interativa, a atividade da prática de voo. Por meio da utilização destes simuladores, os primeiros contatos dos aprendizes com o ambiente de pilotagem puderam ser estabelecidos ainda em terra, diretamente no computador, reduzindo o volume de horas de voo necessárias para a formação do piloto, o que impacta na redução dos custos de treinamento e no aumento da segurança.

Em geral, a evolução dos simuladores ao longo dos anos foi focada no realismo operacional e visual, tanto das aeronaves, quanto do ambiente externo. Foram desenvolvidos, inclusive, dispositivos que simulam cabines completas de aeronaves, ampliando a imersão dos aprendizes por meio do alto nível de realismo alcançado na prática da pilotagem de determinada aeronave.

No entanto, como o foco evolutivo permaneceu na precisão operacional e qualidade visual, a área pedagógica do ensino da pilotagem de aeronaves não fez parte do escopo de desenvolvimento dos simuladores. Isto faz com que as etapas de prática em simulador dos cursos de formação de pilotos profissionais tenham de ser acompanhadas de forma direta e individual pelos tutores, que supervisionam os procedimentos dos aprendizes e analisam seu desenvolvimento prático. $\mathrm{O}$ acompanhamento individual da prática em ambiente de simulação limita o alcance pedagógico dos tutores e, com isso, aumenta seu volume de trabalho, reduzindo a possibilidade de implementação de estratégias pedagógicas que impactem simultaneamente em todo o grupo de aprendizes.

Buscando uma alternativa computacional para a demanda pedagógica dos simuladores, apresentamos a proposta de uma arquitetura pedagógica que permita aos tutores a confecção de assistentes de aprendizagem sintéticos para interagirem com o aprendiz no momento de sua prática no simulador de voo.

\section{Fundamentação Teórica}

Antes da execução do primeiro voo, acreditava-se que uma aeronave motorizada seria um dispositivo simples de controlar e que exigiria poucas habilidades de controle do piloto. Em pouco tempo, foi constatado que esta suposição estava incorreta, uma vez que as primeiras aeronaves possuíam características únicas e fatais. A necessidade da preparação efetiva de pilotos para as tarefas de voo levaram à ideia de criar dispositivos que poderiam simular as características de uma aeronave, o que seria inestimável como ferramenta de treinamento. Esta foi a concepção da simulação de voo. (Lawn, 1998)

\subsection{A simulação de voo}

Segundo Gheorghiu (2013), a simulação é um método usado na aviação composto por um conjunto de tecnologias que reproduzem a interação homem-aeronave para propósitos de treinamento, avaliação de desempenho, pesquisa e desenvolvimento. Se refere, primeiramente, à criação de representações físicas do terreno para reproduzir elementos técnicos e os comportamentos de uma aeronave sob várias condições e em interação com o elemento humano.

Ruiz, Aguado e Moreno (2014) listam alguns dos principais recursos que quaisquer tipos de simulações possuem: são úteis para tomada de decisões; permitem a avaliação e a previsão de potenciais soluções futuras; permitem a otimização do 
comportamento do sistema e oferecem um método seguro e econômico de treinamento de operadores. Informam, ainda, que o componente de simulação em uma experiência de aprendizagem permite levar pessoas ao tempo ou lugar que seria impossível ou provavelmente inviável de praticar diretamente, incentivando assim a aprendizagem dentro de situações artificiais que podem ser totalmente adaptadas para aprimorar a experiência de aprendizagem. Adicionalmente, os recursos já listados podem ser usualmente complementados com a capacidade dos simuladores de representar e conectar grandes volumes de informações por meio de multimídia, como imagens em alta definição e som ambiente altamente fiel, o que torna a experiência de aprendizagem mais atrativa aos estudantes.

Entretanto, segundo Nählinder, Berggren e Persson (2005), a solução de treinamento mais ideal pode se distanciar um pouco da necessidade por simular o mundo tão realístico quanto possível. Acredita-se que simuladores podem aumentar a eficiência do treinamento dramaticamente quando incorporam abordagens pedagógicas como complemento à fidelidade. Ao fornecer uma série de recursos pedagógicos que estão de acordo com a cultura de treinamento, um simulador de treinamento pode provavelmente ser muito mais eficiente do que um simulador com fidelidade quase perfeita.

A partir desta premissa, podem ser propostas soluções como os sistemas associados autônomos, descritos por Levi et al. (1992), que auxiliam um piloto humano a realizar uma missão, por meio da obtenção e compilação de informações, recomendação de ações e, em alguns casos, desempenhando determinadas tarefas da missão.

\subsection{A abordagem pedagógica}

Freitas e Neumann (2009) informam que, por causa do imediatismo e do apelo amplo de aprendizagem em mundos imersivos, o uso de modelagem e simulações computacionais para treinamento estão induzindo uma reconsideração de como os indivíduos aprendem, onde aprendem, o que aprendem e quando aprendem. Isto está levando à revisão das abordagens de aprendizagem, tanto para contextos formais e informais, quanto para contextos mistos. Porém, apesar de muitos aprendizes estarem usando estas ferramentas de forma diária, tem havido muito pouca consideração de mudanças nos modelos pedagógicos que sustentam essas novas formas de organizar a aprendizagem.

Como possíveis estruturas que abranjam essas mudanças em modelos pedagógicos, Carvalho, Nevado e Menezes (2005) propõem as arquiteturas pedagógicas, que são estruturas de aprendizagem compostas pela abordagem pedagógica, software, internet, inteligência artificial, educação à distância e concepção de tempo e espaço. Acolhem didáticas flexíveis, maleáveis e adaptáveis a diferentes temas. As perspectivas de tempo e espaço de aprendizagem passam a se moldar aos ritmos impostos pelo sujeito que aprende, removendo a exigência pela localização em escola ou sala de aula. As arquiteturas funcionam como mapas, mostrando diferentes possibilidades de se realizar algo, fornecendo ao aprendiz a escolha dos caminhos a serem percorridos. Os professores são essenciais para a criação e reinvenção dessas arquiteturas pedagógicas. Já os aprendizes atuam como protagonistas, agindo e refletindo sobre experiências com a orientação do professor. 


\section{Trabalhos Correlatos}

A assistência de aprendizagem em simuladores é composta principalmente por missões ou tutoriais pré-instalados nos simuladores de voo. Os assistentes são procedimentos baseados em passo a passo, de modo a orientar o aprendiz a concluir um passo para que o próximo seja apresentado. A seguir, são descritos estes assistentes pré-configurados em dois dos principais simuladores de voo do mercado: Microsoft Flight Simulator X e FlightGear.

\subsection{Assistentes de Aprendizagem do Microsoft Flight Simulator X - Missões}

O Microsoft Flight Simulator X (FSX) é um dos simuladores de voo comerciais mais tradicionais do mercado. Segundo Lackey (2006), o atrativo principal desta versão do simulador é um módulo de criação de missões poderoso e simples de usar, utilizado para elaborar as 50 missões que vêm embarcadas no jogo. As missões embarcadas vão desde as missões relativamente simples, como procurar por um campista perdido em montanhas nevadas, às mais desafiadoras, em tarefas frenéticas como resgatar trabalhadores de uma plataforma de petróleo explodindo. Segundo Stark (2008), esse módulo fornece uma oportunidade de ensinar habilidades e explorar os recursos e efeitos do FSX de uma maneira desafiadora e divertida.

Os assistentes adotados no FSX atendem à demanda de um instrutor que deseje configurar práticas procedimentais para seus aprendizes. Ainda que os instrutores tenham flexibilidade na configuração das missões, essa abordagem não é melhor por não permitir que o aprendiz seja o protagonista em seu processo de aprendizagem, ou seja, no desenvolvimento de sua aprendizagem por meio de sua própria escolha de caminhos. Pelo contrário, cada passo do procedimento configurado pelo tutor é requerido para a conclusão da missão.

\subsection{Assistentes de Aprendizagem do FlightGear - Tutoriais}

FlightGear é um simulador de voo livre, desenvolvido cooperativamente através da internet por um grupo de entusiastas de simulação de voo e de programação. $O$ FlightGear contém um sistema de tutorial em voo, onde um instrutor simulado fornece lições virtuais. Estas lições variam entre aeronaves, desde simples tutoriais ensinando como iniciar os motores em uma aeronave, até lições completas ensinando como voar pela primeira vez. Cada tutorial consiste em um número de passos discretos que o aprendiz precisa completar. $\mathrm{O}$ instrutor simulado fornece as orientações de como completar cada passo, e observar como o aprendiz o realizou, fornecendo instruções adicionais caso necessário. (Basler et al., 2015)

Os assistentes pré-configurados no FlightGear funcionam de forma semelhante às missões do FSX, descritas anteriormente. O FlightGear também disponibiliza aos instrutores a possibilidade de criação de novos tutoriais. Porém, uma vez que esses tutoriais possuem a mesma limitação das missões do FSX, sendo procedimentos pré-configurados requerendo a execução de uma sequência de passos de forma ordenada, o protagonismo do aprendiz na formação de sua aprendizagem ainda não é alcançado de forma plena. 


\section{A Arquitetura Pedagógica para Assistência de Aprendizagem Prática de Pilotagem de Aeronaves em Simulador de Voo}

No início dos cursos de formação de indivíduos para pilotagem de aeronaves, é requisito que o aprendiz construa um conhecimento teórico a respeito das características do voo, regulamentos de tráfego aéreo, meteorologia aeronáutica, conhecimentos técnicos e motores, além de legislações aeronáuticas, práticas de segurança de voo, regulamentação, medicina e direito aeroespacial. A partir do momento em que o aprendiz conclui sua formação teórica inicial, o mesmo já tem base de conhecimento suficiente para iniciar seu processo de aprendizagem por meio da prática na operação de aeronaves em simuladores de voo.

Uma vez que o aprendiz esteja na etapa de prática em simulador de voo, cabe ao tutor humano a tarefa de supervisionar essa prática. Este processo de supervisão consome tempo e limita a atuação e abordagem pedagógica do tutor. Dadas as particularidades na construção do conhecimento prático de cada aprendiz, a supervisão do tutor humano tem de ser individual. A possibilidade da configuração de assistentes tradicionais no modelo procedimental, como descrito no capítulo anterior, auxilia o tutor na aplicação de exercícios práticos em determinado grupo de aprendizes. Porém, esses assistentes limitam o protagonismo dos aprendizes na formação de sua aprendizagem, pois toda a evolução de determinado exercício prático já foi pré-determinado pelo tutor, exigindo-se que cada passo seja seguido exatamente como previsto para que o próximo passo seja alcançado.

Para a criação de um ambiente que favoreça o protagonismo do aprendiz na formação de sua aprendizagem prática em simulador de voo, é considerada na arquitetura pedagógica uma abordagem flexível, na qual o aprendiz tenha liberdade na realização de sua prática, sendo supervisionado pelo assistente de aprendizagem configurado pelo tutor. Existem outras abordagens pedagógicas possíveis, porém, dada a característica procedimental intrínseca no domínio do conhecimento da operação de aeronaves, entende-se como válido, além de aderente à expectativa atual dos tutores, o uso dos assistentes de aprendizagem enquanto supervisores da prática do aprendiz no simulador de voo.

\subsection{Os Assistentes de Aprendizagem}

Os assistentes de aprendizagem da arquitetura pedagógica proposta são configurados pelos tutores no formato de procedimentos compostos por ações e decisões. Em cada ação ou decisão, podem ser consideradas ou validadas as propriedades de voo em determinado momento da prática do aprendiz. Com base nessas propriedades e nos passos configurados no assistente, o sistema analisa a prática do aprendiz e verifica se ele cumpriu ou não os critérios de determinada decisão. A arquitetura pedagógica possibilita ao tutor que aplique uma abordagem pedagógica flexível, na qual, caso o aprendiz não cumpra determinado passo, o assistente não imponha a ele a necessidade pelo cumprimento, mas sim questione a razão que o levou a tal atitude, permitindo ao aprendiz a escolha do caminho de sua aprendizagem.

Os procedimentos dos assistentes de aprendizagem são configurados visualmente por meio de fluxogramas, nos quais os tutores podem criar as ações e 
decisões e vinculá-las no diagrama, conforme exemplo ilustrado na Figura 1, sendo as ações representadas pelos retângulos, as decisões representadas pelos losangos, o início e fim do assistente representados pelas elipses e os vínculos representados pelas setas. Nessa ilustração, após o início de sua execução, o assistente verifica se o motor da aeronave está ligado. Caso o aprendiz não ligue o motor dentro de 5 minutos, o assistente questiona o aprendiz sobre o motivo. Caso a resposta do aprendiz seja coerente, como, por exemplo, se ele ainda estiver realizando uma checagem pré-voo, o assistente volta ao início da contagem e verificação do motor ligado. Caso a resposta recebida não proceda, o assistente questiona novamente ao aprendiz sobre o motivo do motor ainda não estar ligado, até que a resposta seja coerente, ou até que o aprendiz ligue o motor. Caso o aprendiz ligue o motor, o assistente de aprendizagem de decolagem passa a analisar se a aeronave já atingiu a altitude de cruzeiro. Enquanto não atingir, são verificadas outras propriedades de voo, com os devidos questionamentos específicos. Caso atinja, o assistente de decolagem é finalizado.

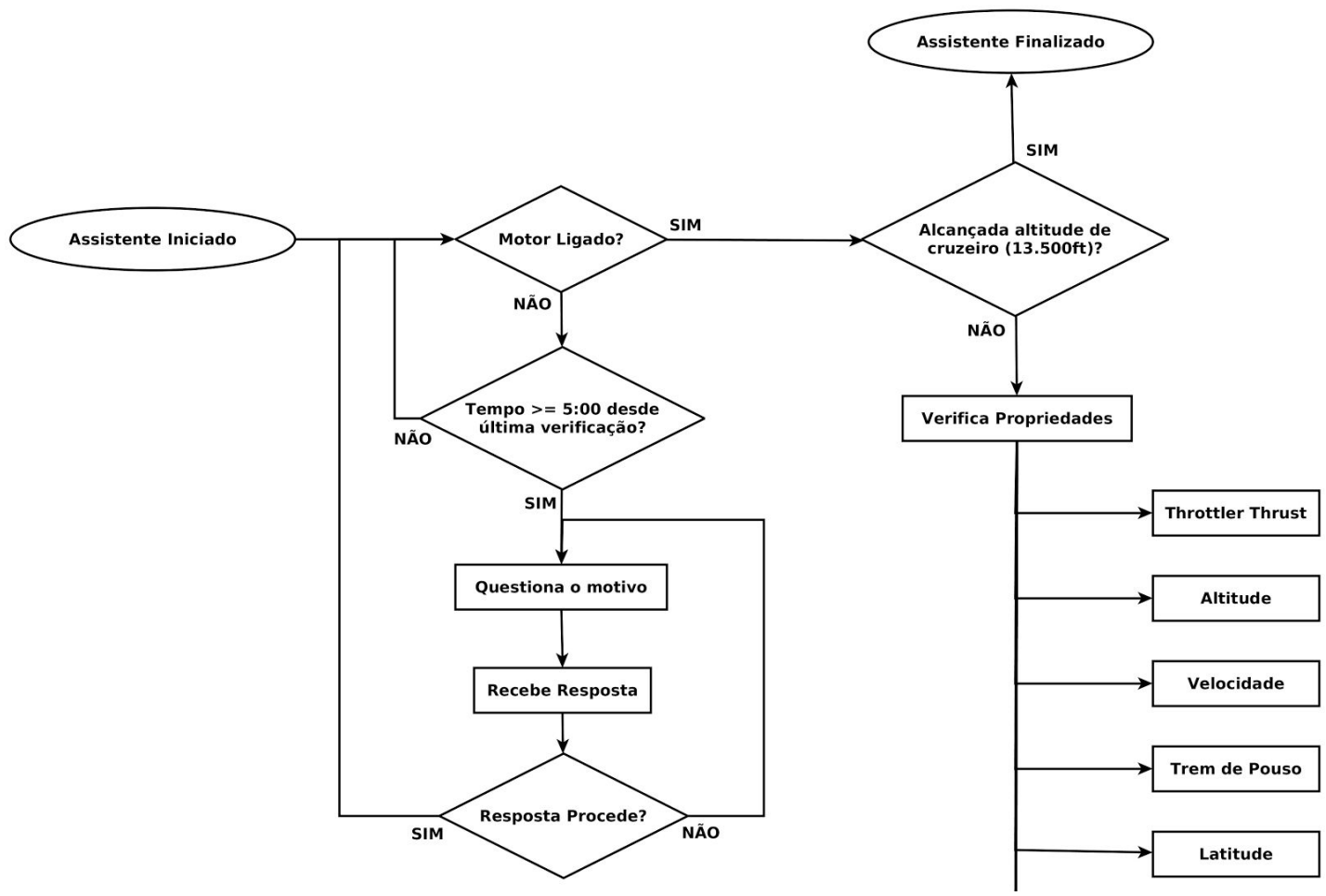

Figura 1. Parte inicial de um possível assistente de aprendizagem do procedimento de decolagem.

O tutor tem autonomia para configurar quantos assistentes forem necessários para determinada prática no simulador. $\mathrm{O}$ aprendiz pode, por exemplo, estar em uma prática de voo livre no simulador e o tutor configurar um assistente de aprendizagem que, em determinado momento, altere as propriedades de voo, de modo que surja fogo em um dos motores da aeronave. Nesse momento, o aprendiz tem que lidar com a situação emergencial e o assistente pode auxiliá-lo questionando quais são os passos que o aprendiz irá realizar e qual a razão para a escolha desses passos. O tutor pode configurar um procedimento padrão para solução emergencial desse problema, e compará-lo com as ações escolhidas pelo aprendiz. Como forma de garantir o protagonismo do aprendiz na formação de sua aprendizagem, o assistente não intervém 
nas ações do aprendiz, nem mesmo demonstrar o caminho a ser seguido a ele, mas sim observa as ações do mesmo e questionar seus motivos, armazenando suas respostas. Caso as ações/decisões adotadas pelo aprendiz sejam mais eficientes do que as pré-configuradas pelo tutor, o sistema pode aprendê-las e apresentá-las ao tutor como melhorias para o assistente de aprendizagem. Essa eficiência pode ser medida com respeito a alguns critérios, como tempo de manobra, consumo de combustível, etc.

\section{Estrutura Computacional}

Para o uso dessa arquitetura pedagógica, em um grupo de estudantes de cursos de formação de pilotos de aeronaves, o tutor precisa de um ambiente que centralize a configuração dos assistentes e das simulações a serem realizadas, além de integrar múltiplas estações de simulação, de forma simultânea ou não. Adicionalmente, caso o tutor deseje acolher uma didática flexível, adaptando-se ao espaço e tempo do aprendiz, conforme a proposta desta arquitetura pedagógica, é necessária uma estrutura distribuída via internet.

A integração dos assistentes de aprendizagem é suportada por um sistema distribuído, sob uma infraestrutura composta por agentes de software executando em um servidor central, na estação de simulação e em um dispositivo móvel pessoal do aprendiz. A integração desses agentes de software é feita via internet. A Figura 2 ilustra a arquitetura dos agentes de software integrados via internet. Essa infraestrutura da arquitetura pedagógica é descrita a seguir.

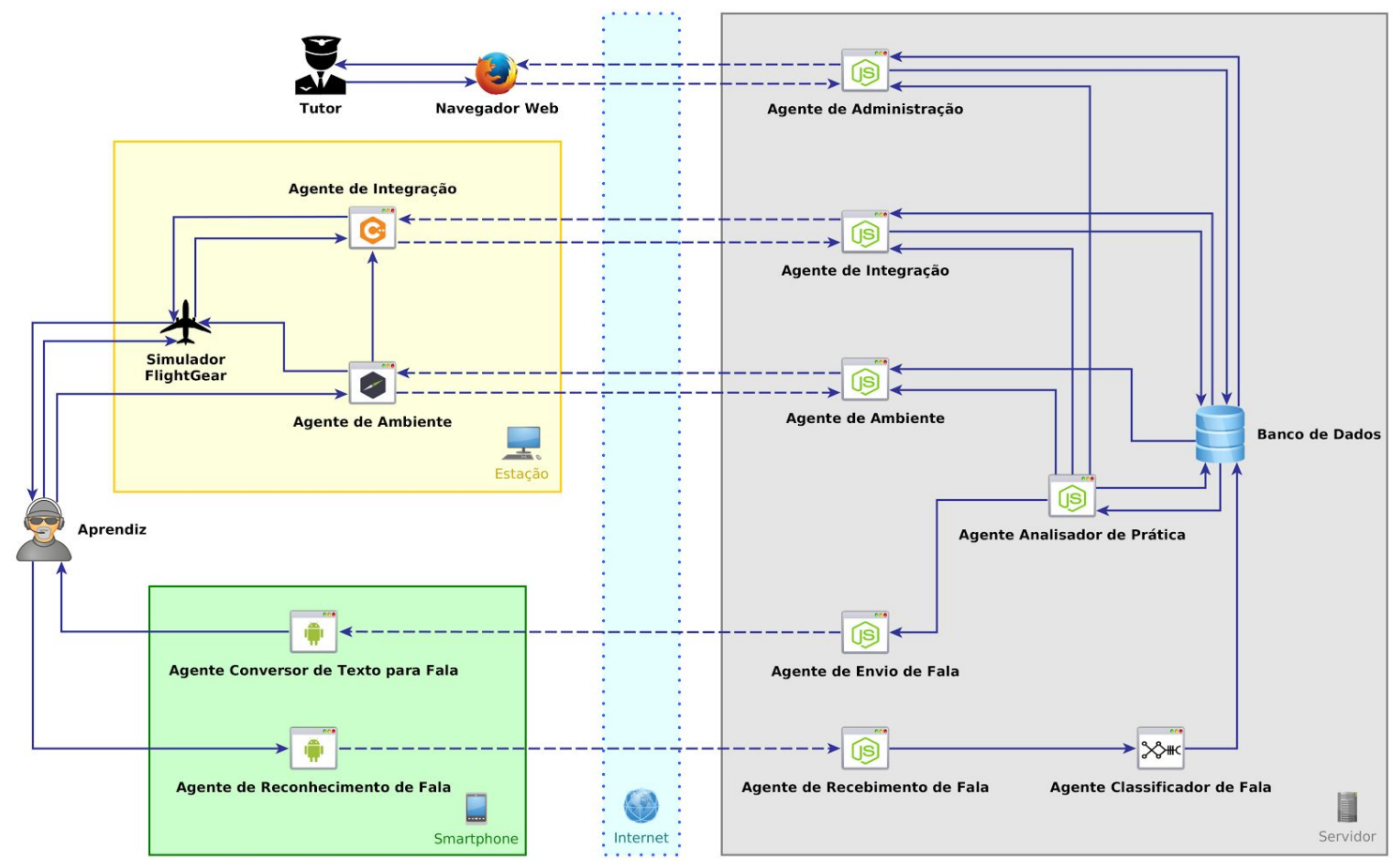

Figura 2. Infraestrutura da arquitetura pedagógica como um sistema distribuído com agentes de software integrados via internet.

O tutor acessa o agente de administração, localizado no servidor, por meio do navegador web. Com o agente de administração o tutor pode criar novos assistentes de aprendizagem e ambientes de simulação, como, por exemplo, a aeronave a ser utilizada, 
em qual aeródromo é iniciada a simulação, data e hora, quais as condições meteorológicas no momento, entre outros. O agente de administração armazena esses novos assistentes de aprendizagem e ambientes de simulação no banco de dados.

O aprendiz acessa o agente de ambiente na estação para iniciar sua simulação. $\mathrm{O}$ agente de ambiente na estação acessa o agente de ambiente no servidor para capturar os dados de configuração da simulação a ser executada. Ao capturar os dados da simulação, o agente de ambiente na estação executa o simulador FlightGear parametrizado com a simulação específica para o aprendiz. A partir deste momento, o aprendiz inicia a prática de simulação e interage com o FlightGear. O simulador FlightGear foi escolhido por possuir código-fonte aberto, ser amplamente documentado e possuir uma linguagem de integração robusta.

Uma vez que o aprendiz inicie sua prática no FlightGear, o agente de integração na estação inicia o processo de captura das propriedades de voo e envio para o agente de integração no servidor. $\mathrm{O}$ agente de integração no servidor recebe as propriedades e as armazena no banco de dados.

O agente analisador de prática lê as propriedades armazenadas no banco de dados e as analisa junto aos passos configurados pelo tutor nos assistentes de aprendizagem. Assim que necessário, o agente analisador de prática envia interações em fala, por meio do agente de envio de fala, além de interações no simulador, por meio do agente de integração.

O agente conversor de texto para fala do smartphone do aprendiz captura os textos enviados pelo agente de envio de fala do servidor e os converte para áudio, conversando diretamente com o aprendiz. Caso o aprendiz responda via fala a interação recebida, o agente de reconhecimento de fala do smartphone transforma essa fala em texto e o envia para o agente de recebimento de fala do servidor. O agente de recebimento de fala encaminha os textos para o agente classificador de fala, que identifica os padrões e armazena a interação do aprendiz no banco de dados. Caso o aprendiz responda via comandos no simulador FlightGear, os agentes de integração na estação e no servidor armazenam essas ações.

O agente analisador de prática realiza a análise do padrão da resposta recebida do aprendiz, seja via fala ou comandos no simulador, e verifica se a ação realizada está compatível com o procedimento configurado no assistente de aprendizagem. Caso a ação do aprendiz resulte em falha catastrófica, o agente analisador de prática envia um comando ao agente de ambiente do servidor para que reinicie a simulação no agente de ambiente da estação.

\section{Perspectivas Futuras e Considerações Finais}

A implementação da arquitetura pedagógica proposta neste trabalho pode auxiliar os tutores de cursos de formação de pilotos de aeronaves na utilização de uma abordagem didática flexível para a prática em simuladores de voo. Por meio da configuração dos assistentes de aprendizagem, o tutor pode estabelecer de modo detalhado seus objetivos didáticos para determinado grupo de aprendizes e aplicá-los, caso necessário, simultaneamente para o grupo inteiro.

A arquitetura pedagógica apresentada é flexível por ser composta por recursos 
digitais que disponibilizam ao tutor as ferramentas necessárias para que ele planeje a abordagem específica para cada prática no simulador de voo. Ainda que o foco dessa arquitetura esteja no protagonismo do aprendiz, diante a formação de sua aprendizagem, cabe ao tutor determinar se determinado procedimento do assistente de aprendizagem deve ser seguido integralmente de acordo com o configurado. A garantia da autonomia do tutor, durante a configuração dos assistentes de aprendizagem, viabiliza o uso da arquitetura pedagógica proposta, independente do método didático determinado pelo tutor ou pela instituição de ensino, para a prática em simulador de voo.

Esta arquitetura pedagógica pode, inclusive, viabilizar a existência de disciplinas a distância, para as simulações de voo dos cursos de formação de pilotos de aeronaves. Uma vez que os tutores não precisam acompanhar fisicamente e individualmente os aprendizes em sua prática, pois os assistentes de aprendizagem são previamente configurados, torna-se viável a adoção da metodologia não presencial durante as simulações. A viabilização dessa etapa em ambiente não presencial, aliada às etapas teóricas dos cursos de formação neste mesmo formato, facilita a formação de novos pilotos de aeronaves, pois reduz a necessidade da presença física dos tutores e permite que os estudantes escolham tanto o local quanto o tempo determinado para a realização dos estudos, uma vez que podem utilizar seus próprios computadores para as etapas iniciais de simulação.

Etapas de prática que utilizem simuladores de voo completos, que simulam toda a cabine da aeronave, por estarem localizados nas instalações das instituições de ensino, exigem a presença física dos aprendizes, o que reduz a aplicabilidade da infraestrutura distribuída do sistema. Entretanto, a flexibilidade fornecida aos tutores na configuração dos assistentes de aprendizagem e a promoção do protagonismo do aprendiz validam a proposta da arquitetura pedagógica apresentada.

Para que esta proposta de arquitetura pedagógica seja amplamente usada e validada, ela será oferecida, sem custos, para instituições de ensino civis e militares que ofertem cursos de formação de pilotos de aeronaves. Essa oferta é possível uma vez que o simulador de voo FlightGear é gratuito e não possui restrições de uso em sua licença.

A adequação da abordagem pedagógica das etapas de prática em simulador dos cursos de formação de pilotos de aeronaves, com os avanços tecnológicos atuais, pode fornecer, tanto aos tutores quanto aos aprendizes, uma experiência de aprendizagem mais consistente e flexibilizar as estruturas didáticas atualmente em vigência.

A adoção da arquitetura pedagógica proposta pode, num futuro próximo, aliada com as tecnologias de ensino à distância já estabelecidas, possibilitar a existência de cursos de formação de pilotos de aeronaves cuja presença física dos aprendizes só seja exigida na última etapa do curso: na prática de voo realizada diretamente na aeronave.

\section{Referências}

Basler, M. et al. (2015) "The FlightGear Manual - For FlightGear version 3.4.0", http://mapserver.flightgear.org/getstart, Março.

Carvalho, M.J.S., Nevado, R.A. e Menezes, C.S. (2005). “Arquiteturas pedagógicas para educação à distância: concepções e suporte telemático", Anais - XVI Simpósio Brasileiro de Informática na Educação, 1, 362-372. 
CBIE-LACLO 2015

Anais dos Workshops do IV Congresso Brasileiro de Informática na Educação (CBIE 2015)

Gheorghiu, A. (2013) "Flight Simulation in Air Force Training. A Knowledge Transfer Efficiency Perspective”, In: Journal of Defense Resources Management, vol. 4, p. 153-158, Transylvania University of Brasov.

Lackey, J. (2006) "Microsoft Flight Simulator - Flying the friendlier skies", In: Computer Gaming World, issue 267, p. 64-64.

Lawn, P. (1998) "The Enhancement of a Flight Simulator System with Teaching and Research Applications", Dissertação de Mestrado, The Department of Mechanical Engineering, Concordia University, Montreal, Quebec, Canada.

Levi, K.R. et al. (1992) "An Explanation-Based-Learning Approach to Knowledge Compilation - A Pilot's Associate Application”, In: IEEE Expert, vol. 7, issue 3, p. 44-51.

Nählinder, S., Berggren, P. e Persson, B. (2005) "Increasing Training Efficiency Using Embedded Pedagogical Tools in a Combat Flight Simulator", In: Proceedings of the Human Factors and Ergonomics Society 49th Annual Meeting, p. 2197-2200.

Ruiz, S., Aguado, C. e Moreno, R. (2014) "Educational Simulation in Practice: A Teaching Experience Using a Flight Simulator", In: Journal of Technology and Science Education, vol. 3, p. 181-200, Barcelona, Espanha.

Stark, P. (2007) “FSX Mission Building - Tools of the Trade”, In: PC Pilot, issue 48, p. 40-43. 